\title{
A Practical Approach of Different Programming Techniques to Implement a Real-time Application using Django
}

\author{
By Sebastian Stigler ${ }^{*} \&$ Marina Burdack
}

Real-time web-applications surround our daily life. The most popular are shopping platforms like Amazon or Zalando. Behind these websites huge and complex shop-systems are typically used. These systems manage and handle thousands of parallel synchronous and asynchronous tasks like user interactions, user authentications, communication with an ERP system (enterprise resource planning), or the handling of the website visualization in the background. Through the independence of the computer system and the portability on other devices, like touch panels or mobile phones the web-technology is also used for industry 4.0 applications. The applications can be e. g. predictive maintenance dashboards or monitoring dashboards for the production line. The key task for these dashboards are the visualization and analyzing of a huge amount of data in real-time. For both scenarios, the data have to be preprocessed continuously in different steps. In summary, the industry 4.0 application has to manage a different kind of tasks compared to a typical webshop. These tasks contain for example big data preprocessing tasks, which are mostly CPU bound, in contrast to the typically I/O bound task of a webshop (e.g. database access). The main problem with web application frameworks is that they are not designed to execute a lot of CPU bound tasks and handle webrequests at the same time. The purpose of this paper is to compare two different programming techniques for the work with CPU bound tasks in a real-time web application for the use in industry 4.0 without the use of third-part software like Spark. The research employed two approaches: on the one hand multiprocessing and on the other hand task queues. In this work, two kinds of web-applications on the base of python's web framework Django are implemented. The first application uses multiprocessing and the second application uses the Celery task queue to organize different tasks. The general building blocks are the sensor data streaming via MQTT and the data transformation in various steps. The result of this transformation can be used to visualize the data in real-time charts for example. The implemented web-applications are evaluated by the following criteria:

- Performance while working with a huge amount of streaming data.

- Possibilities to optimize the load distribution and avoid overloading.

- Possibilities to scale up and down hardware.

Keywords: Django, Machine Learning, Multithreading, Real-Time-Application, Task Queue.

"Research Fellow, Aalen University of Applied Sciences, Germany.
*Research Fellow, Aalen University of Applied Sciences, Germany. 


\section{Introduction}

Real time web applications surround our daily life. Different web applications can be found in different areas like the web shops or google, but also in different areas like the industry or the medical sector. Both sectors work with real time streaming data. In the industry 4.0 often monitoring dashboard are built, which analyze sensor data from machines for example for predictive maintenance or for quality prediction (Burdack and Rössle 2019, Rössle and Kübler, 2017). In the medical sector, web applications are used for the tracking of medicine intakes for example (Vinjumur et al. 2010).

But all these different applications consist of more than one component. All these applications need a database or a data storage, their own business logics, user authentication and so on. But the highest influence on the performance of the web applications has the streaming data. In industry 4.0 settings there is a big amount of sensor data, which need to be processed and stored in the application for further analytics or machine learning purpose. All these approaches follow the standard CRISP model (Wirth 2000), where a central point is the data preparation or in the field of machine learning it is called data preprocessing. These approaches are the typical working areas of data scientists.

The most popular programming language in Data Science for data analysis and web development is python (Kaggle, et al. 2018, Python Software Foundation and JetBrains 2018). According to Python Software Foundation and JetBrains (2018) Django and Flask are the most popular web frameworks in python.

But the program language and a web framework are not enough for a realtime application. Especially for working with a big amount of data, there is often third party software ${ }^{1}$ like Spark (Meng et al. 2016), Hadoop (Borthakur et al. 2011) or AirFlow (Kotliar et al. 2018) needed to scale the application, and thus to schedule the working tasks.

Nevertheless, all these tools only work with good performance, if they are configured by data scientists with long experience in using them. But these experiences are often not available in young data scientist teams or the effort for the implementation for a small project is too high.

For this research work, a Django application was developed, which allows to configure and run different preprocessing tasks efficiently. In addition, our implementation allows to configure and run machine learning tasks too, without any changes. Only the different machine learning tasks have to be implemented and registered in the application database. Through the strict definition of tasks, different data processing pipelines can be modeled with this approach beginning with the configuration of the data source (MQTT, streaming database ...) and ending with the visualization or presentation of the results. All can be done in one application.

This article focuses on the most important part in the machine learning pipeline: the preprocessing of data. The findings and results of this approach are

\footnotetext{
${ }^{1}$ Which are not written in python.
} 
used in the implementation of the whole framework for machine learning in Burdack and Rössle (2019). This leads to the following research questions:

- Is it possible to implement the preprocessing part of the machine learning pipeline in an industry 4.0 real-time web application, without the use of (non python) third party software in python?

- How can you scale the application in an easy way, if the amount of incoming data increases significantly?

\section{Literature Review}

In literature different approaches and software solutions exist to develop software applications, which work efficient and are scalable.

The most popular software for scaling software applications, especially in the field of big data and machine learning are Apache Spark (Meng et al. 2016) (https://spark.apache.org/), and Hadoop (Borthakur et al. 2011).

"Apache Spark is a popular open-source platform for large-scale data processing that is well-suited for iterative machine learning tasks" (Meng et al. 2016). It consists of different application parts. The core application is Apache Spark, which can be extended with different libraries to a powerful data analytics engine. The different libraries are Spark SQL, Spark Streaming, MLib and GraphX. ${ }^{2}$ Spark SQL adds interactive queries and Spark Steaming the handling of Streaming Data. The machine learning library of Spark MLib contains predefined machine learning algorithm like logistic regression or decision trees, but the number of algorithms is less than the number of algorithms in the popular machine learning library scikit-learn for python (Pedregosa et al. 2011). The GraphX library completes the Apache Spark Framework, but only allows to build "graph from a collection of vertices and edges." 3

For our research approach, Apache Spark is not useful, because it is not a native python framework but a framework written in scala with python bindings (Karau 2015, 9).

"The Apache Hadoop software library is a framework that allows for the distributed processing of large data sets across clusters of computers using simple programming models. It is designed to scale up from single servers to thousands of machines, each offering local computation and storage." The programming language for Hadoop is Java; this is not useful for our research (Lam 2010).

The last relevant project from the apache family is Apache Airflow (Kotliar et al. 2018). "Airflow is a platform to programmatically author, schedule and monitor workflows." As programming language python is used. But: "Airflow is not a data streaming solution. Tasks do not move data from one to the other

\footnotetext{
${ }^{2}$ https://spark.apache.org/.

${ }^{3}$ https://spark.apache.org/docs/latest/graphx-programming-guide.html\#graph-builders.

${ }^{4}$ https://hadoop.apache.org/.

${ }^{5}$ https://airflow.apache.org/.
} 
(though tasks can exchange metadata!)." "Thus is it not useful for our approach, because in the preprocessing tasks it is unavoidable to move the prepared data from one task to the next.

Besides the big software solutions from Apache, there are a few solutions like Celery, TaskTiger or Parsl which work native with Python and are available as Python packages.

"Celery is an asynchronous task queue/job queue based on distributed message passing. It is focused on real-time operation, but supports scheduling as well. The execution units, called tasks, are executed concurrently on a single or more worker servers using multiprocessing." Because of these key characteristics, Celery is of main interest for our research.

An alternative for Celery is TaskTiger, which is also a task queue. But the library is still in development and no major release is available (current version 0.10.1) ${ }^{8}$ Therefore, it will not be used in this research.

Babuji et al. (2018) describe Parsl (Parallel Scripting Library) ${ }^{9}$ in their paper. Parsl is a library that "can be easily integrated into Python-based gateways" (Babuji et al. 2018). This library allows a simple management and workflow scaling. Its main purpose is to "manages the execution of the script on clusters, clouds, grids, and other resources" (Babuji et al. 2018). It "orchestrates required data movement; and manages the execution of Python functions and external applications in parallel" (Babuji et al. 2018). The authors do not implement a web application but concentrate on the parallel execution of other programs. Furthermore, there is no example implementation and no description on how to distribute tasks in Parsl.

Lunacek et al. (2013) describe in their paper an approach about "the scaling of many-task computing" on cluster supercomputers (Lunacek et al. 2013). They compare the python libraries IPhython parallel and Celery. For their approach they evaluated both tools on a cluster. This is an interesting approach, but in our approach we are focusing on small settings like a laptop or a workstation as well as big settings like clusters.

\section{Methodology}

Here we will describe what kind of scaling options Python offers for the implementation of the data processing component of the web application. We will also describe which of these options we have implemented and what architectural choices we made. Finally we present the mathematical foundations we use to determine the scaling of the application.

\footnotetext{
${ }^{6}$ https://airflow.apache.org/.

${ }^{7}$ http://www.celeryproject.org/.

${ }^{8}$ https://pypi.org/project/tasktiger/.

${ }^{9} \mathrm{https} / / /$ parsl-project.org/.
} 


\section{Handling the Data Processing in the Web Application}

In order to share the resources of our computer between the web application and the processing of the date we receive from the data source, we need to discuss, which options Python provides to solve this issue. Furthermore, if the load on the computer to process the data becomes too high, we need to distribute the computations among different servers. Generally there are three options available:

- Implement the data processing in the same thread as the web application (i.e. single threaded approach).

- Use a concurrency model Python provides.

- Use distributed task queue to share the computation between multiple servers.

\section{The Single Threaded Approach}

This programming model is not recommended because the application can at any time either handle a web request or a data processing task but not both at the same time. Depending on how fast new data arrives and how computationally intensive the processing is, users may experience a noticeable delay in the handling of their web request.

\section{Concurrency Models of Python}

To determine which concurrency model is appropriate, we have to determine if the data processing tasks are CPU bound or I/O bound tasks.

A CPU bound task utilizes the CPU in long bursts with short waiting periods for I/O in between. An I/O bound task in contrast waits most of its execution time for I/O and utilizes the CPU in short burst (Tanenbaum and Bos 2014).

With this characterization we will see later in this article that the data processing tasks are almost always CPU bound.

The Python Standard Library offers the following concurrency models (Fein 2018):

- The threading library provides thread-based parallelism for Python.

- Process-based parallelism is provided by the multiprocessing library.

- With the asyncio library it is allowed to write single threaded concurrent code.

- Finally the concurrent.future library enables us with a high-level interface for asynchronously executing callables.

\section{Thread-based Parallelism}

The threading model in Python is severely restricted because of the global interpreter lock (GIL) (Wouters 2017). Due to this lock Python cannot fully utilize a multiprocessor/multicore system. Two different threads in Python can't run two 
instructions simultaneously because the interpreter has to acquire the GIL before the execution of each command. Hence multithreading in Python is helpful, if the tasks you wish to parallelize are I/O-bound.

Therefor this parallelism model has to be rejected for our usecase, because it would not help the data processing tasks to scale and use the full capacity of a multiprocessor/multicore system.

\section{Process-based Parallelism}

This kind of parallelism has the advantage, that for each process a new Python interpreter is started and each interpreter has its own GIL. Because of this, the full capacity of a multiprocessor/multicore system can be reached by starting as much processes as there are processors respectively core in the processors.

Process-based parallelism is therefore useful in situations where CPU-bound tasks have to run in parallel. Hence we will use this method to research its scaling behavior in the application.

\section{Asynchronous I/O}

asyncio is used as a foundation for multiple Python asynchronous frameworks that provide high-performance network and web-servers, database connection libraries, distributed task queues, etc.

asyncio is often a perfect fit for IO-bound and high-level structured network code. $^{10}$

This characterization also excludes this technique from the scope of this research.

\section{The Concurrent.future Library}

This library provides the ProcessPoolExecutor class which enhances the mutiprocessing library. It starts a pool of processes, manages the communication between the main process and the processes in the pool and starts tasks in the processes of the pool.

As this is exactly the same feature we would have implemented for the execution of the data processing tasks, we will use this class when we examine process-based parallelism for our application.

\section{Distributed Task Queues in Python}

In the Python ecosystem Celery (Solem and contributors 2019) is the defacto standard for distributed task queues. Furthermore Django supports Celery out of the box since Celery version 3.1. ${ }^{11}$

\footnotetext{
${ }^{10}$ First and second paragraph: quote from https://docs.python.org/3/library/asyncio.html.

${ }^{11}$ See $h$ ttp://docs.celeryproject.org/en/latest/django/first-steps-with-django.html.
} 
Celery itself needs a message broker which distributes the tasks from the web application to the worker processes on other servers. The Celery documentation recommends RabbitMQ (Pivotal Software Inc. 2019) or Redis (Sanfilippo and contriburors 2019) as message broker.

From this two message brokers RabbidMQ is the more powerful one (regarding its features and configuration possibilities). Unfortunately this is a disatvantage for our approach because we want to develop an application that needs as less additional knowledge outside of the Python ecosystem as possible.

In contrast Redis is easy to install and needs no further configuration for our purpose. Therefore, we use Redis as message broker for Celery in our application.

\section{Architecture}

After the discussion of the theoretical fundamentals for multiprocessing and distributed task queue with Celery the key results were used to develop the following two different architecture models. Both architecture models need to fulfill the following requirements:

- fast and consisting processing of big amount of data

- possibility for flexible scaling

- after initial configuration a comfortable configuration

- no integration of third-party software, only python libraries are allowed

- possibility to connect different data sources

- avoid data lost

- flexible output of the result data

- both architectures should be integrated in one application

\section{Multiprocessing}

The architecture of multiprocessing contains different components. The data sources can be messages from a MQTT broker, data from a special time series database for sensor data (Burdack et al. 2018), data from a PostgreSQL database, collection of csv-files and so on. For multiprocessing it is not necessary that this data is static, it is also possible to work with streaming data.

The input data - static or streaming data - will be collected in the buffer area in buckets. Each of these buckets contains a predefined number of input data. If this count of input data in the buffer is reached, the whole data package is sent to the preprocessing app. The idea behind the buffering is to provide a method to reduce the arrival rate in the app at the cost of increasing the service time.

The preprocession (web-) app as well as the multiprocessing pool are parts of the same host system. This host system defines the maximum count of workers for the multiprocessing pool depending on the count of processors resp. cores.

The number of workers for the multiprocessing pool in this approach is defined by the preprocessing app. If no limitation will be given, the host system in theory can take the whole available count of cores for the workers. 
The data package which will be sent to the preprocessing app enters the multiprocessing pool. There the processing rules which are defined in the chained task in the preprocessing app will be applied to the input data.

The result of this modification will be sent as one data package to the result interface. The result interface can send these result data to for instance a Bokeh plots, other application for example a machine learning platform or to other host system for further modifications. The whole procedure is presented in Figure 1.

Figure 1. Multiprocessing Architecture

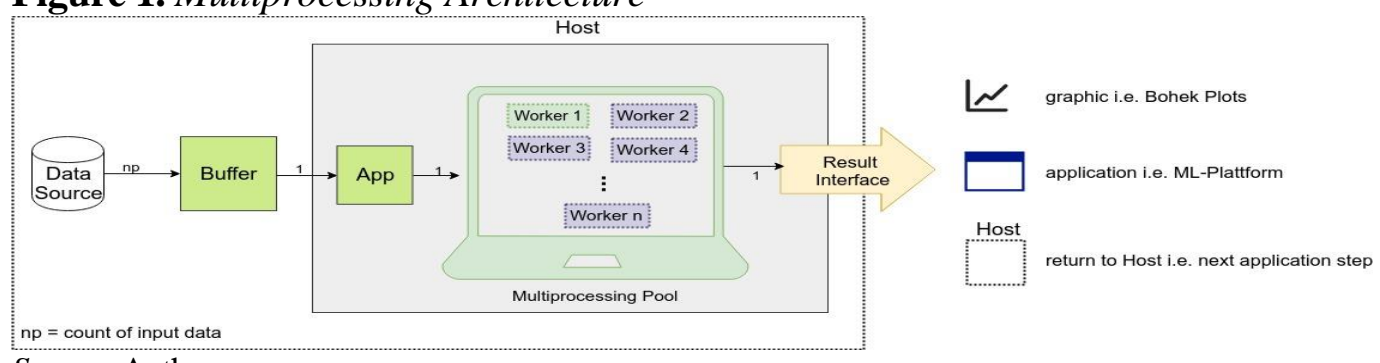

Source: Authors.

The multiprocessing pool works with a special chained task, shown in Figure 2 , which is only proceeded on one worker. The defined package of input data discussed below are json files, which are be compressed and base 64 encoded to reduce the bandwidth and avoid string encoding problems in python. This transformed data package is the input data for the chained task.

The chained task starts with the prepare task, which decodes the data and transforms the raw data into a pandas data frame. This panda data frame is the basis for the processing in the further tasks. All the different defined tasks are based on working with pandas data frames for high-performance data manipulation.

Based on the configuration in the preprocessing app, a specific number of subtasks (Task 1 - Task $\mathrm{n}$ ) have to be processed. The output of each task will be used as the input of the following task. If the last task is finished, the result data frame is passed to the result task.

The result task prepares the data frame for the further working as noted above for example for other applications. The subtasks are monitored and scheduled by a scheduler, which looks after the right execution of the tasks in the right order.

In the multiprocessing approach all the subtasks are executed in the same worker process. No data has to leave the worker process in order to pass the data to the next subtask.

Figure 2. Chained Task in the Multiprocessing Architecture

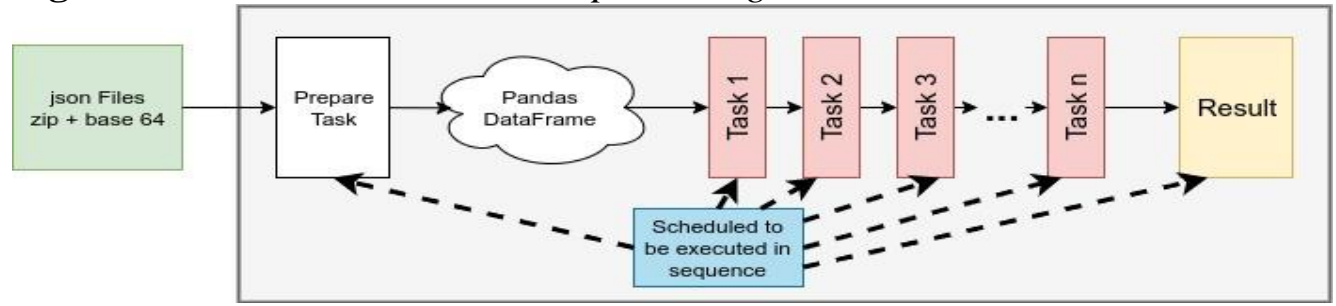

Source: Authors. 


\section{Distributed Task Queue}

The architecture of the distributed task queue with Celery is in contrast to multiprocessing more complex. The data source is the same as describe in multiprocessing above. Here it is also possible to work with different kinds of data sources like streaming or local data. The input data will also be collected until a predefined count of input data in the buffer. The whole collected data package will be sent to the host system, which only contains distribution components of Celery and the preprocessing application. But the data will be not transformed on the host system itself, the data package will be passed on to Redis, which manages the Celery workers on the different worker nodes.

These worker nodes transform the data with the preprocessing rules, which are defined in the application.

If all subtasks are done, the result is passed to the result interface. This result interface can be the same as described in multiprocessing. The whole procedure is shown in Figure 3.

Figure 3. Celery Architecture

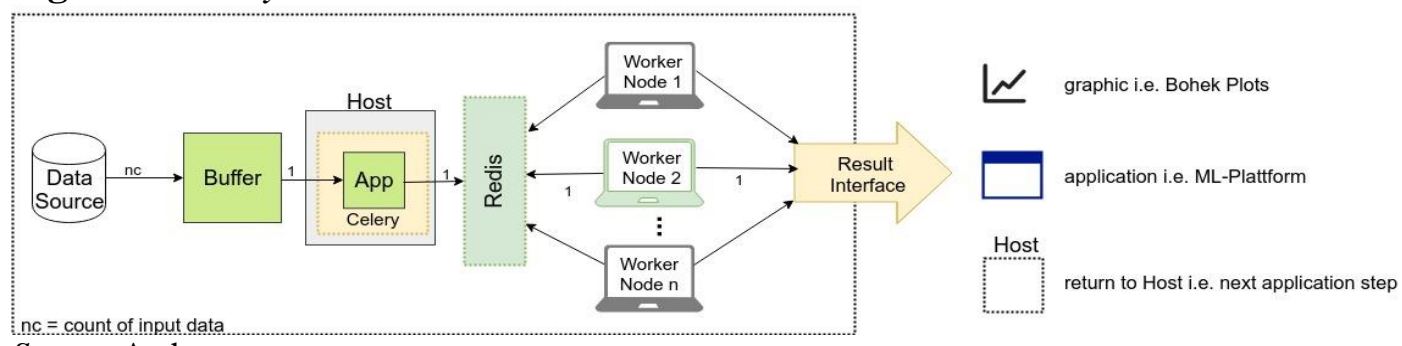

Source: Authors.

The distributed task queue with Celery works with a special chained task, which is visualized in Figure 4.

Figure 4. Chained Task in the Distributed Task Queue Architecture

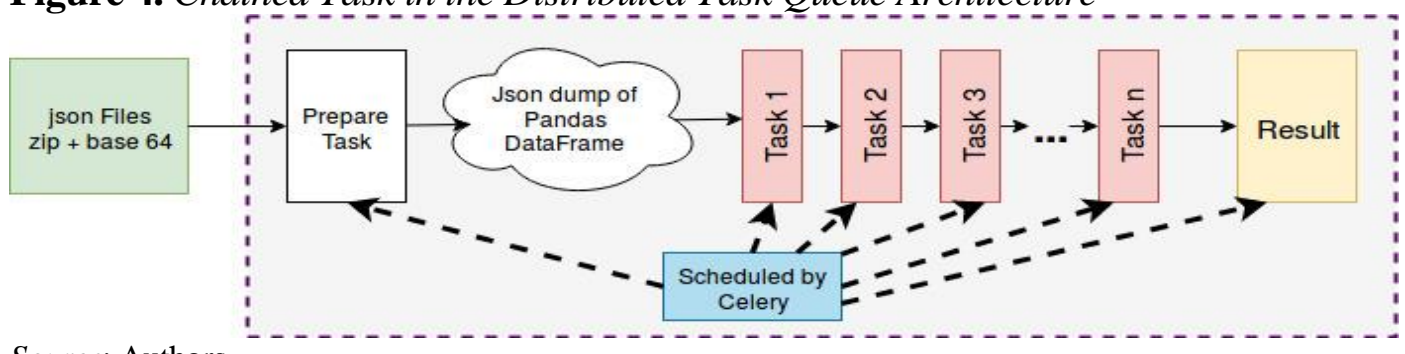

Source: Authors.

In contrast to the multiprocessing architecture, Celery provides the scheduling and subtask chaining (i.e. use the output data of a previous task as input for the next task) out of the box.

Furthermore, Celery distributes the subtasks round robin to the worker processes on the worker nodes. That makes it necessary to exchange the data via Redis among the subtasks. Unfortunately pandas data frames cannot directly be transported via Redis. Therefore we first had to convert the result of a subtask into 
a json representation of the pandas data frame, then send it via Redis to the next subtask and convert it back to a pandas data frame.

This conversion before exchanging data is the reason we introduced the buffer component in the first place. It allows us to reduce the number of conversions per data row significantly.

Application of Queueing Theory

Finally we need a tool to help us to determine how to scale our application. It should consider the following point:

- The arrival rate of the data.

- The tasks chained together to process the data (see Figures 2 and 4).

- The number of worker processes that run the chained task on the data.

This tool can be found in the mathematical field of Queueing Theory by applying the following:

A queue with $c$ servers is stable (will not grow without bound) if the following inequality holds:

$$
\rho=\frac{\lambda}{c \mu}<1
$$

Where $\rho$ is the server utilization, $\lambda$ is the arrival rate and $\mu$ is the service rate (the inverse of the service time for one task) (Bhat 2015).

\section{Assigning the Components of this Inequality to our Application}

We will show how the variables $c, \lambda$ and $\mu$ must be applied to our problem, in order to determine the server utilization $\rho$.

\section{The First Component is the Number of Server c}

In this context server denotes the consumer of the data not the physical computers in our server room.

To explain this term for our purpose, we have to distinguish between the multiprocessing architecture and the distributed task architecture.

\section{$\underline{\text { Multiprocessing Architecture }}$}

In this case $c$ is the number of worker processes in the process pool (see Figure 1). Additionally this number may not exceed the number of processor cores minus one. This spare core is used to serve the http requests to the application. The others are used for the data processing. It is important to not exceed the number of cores on the system because the data processing tasks are CPU-bound. If the 
number of workers is bigger than the number of available cores, then the data processing tasks have to wait for previous tasks to finish.

\section{Distributed Task Queue Architecture}

Here $c$ is the number of all the worker processes summed over all worker nodes (see Figure 3). Analog to the multiprocessing case the number of worker processes per worker node should not exceed the number of processor cores on the respective node.

\section{The Second Component is the Arrival Rate $\lambda$}

The arrival rate denotes how fast new data is ready to be processed, for example, how often a sensor value is read in a given time interval.

We have to distinguish two different arrival rates, depending on where we measure them:

$\lambda_{d s}$ The arrival rate of the data rows measured at the data source.

$\lambda_{\text {buf }}$ The arrival rate of the buffered data rows (messages) measured after the buffer. holds:

If the buffer puts $n$ data rows in one message then the following equation

$$
\lambda_{d s}=\lambda_{b u f} \cdot n
$$

The Third Component is the Service Rate $\mu$

This is the inverse of the time the data processing task takes to calculate its result for a given data row or a buffered message depending on which arrival rate was chosen (see Figures 8-9).

To calculate $\mu$ for the chained task, you should sum over the service time of all the tasks that form the chained task and take the inverse of it.

\section{The Final Component is the Server Utilization $\rho$}

It is the percentage of the utilization of the maximal amount of the computing power of the complete system.

A positive value much smaller than one, denotes that the system could process more data without problems. A value very close to one means, that you use the complete computation power of the system without stalling the output. And a value of one or greater, shows, that your tasks require more computation power than the one your system can provide. In this case the queue with unprocessed data grows faster than it can be consumed and the system becomes unstable. The concrete application of this inequality will be discussed in the findings section. 


\section{Findings}

\section{The Preprocessing App}

Through the architecture of the web framework from Django (Vincent 2018), it is possible to integrate other python modules like MQTT module or database connection modules. This main program structure allows, to implement the process components for multiprocessing and task queue in the same application. Through a simple configuration setting in the app the method can be selected. Through this possibility, no third part integration of other programs are necessary. This leads to an easy managed application, which only depends on python and its modules.

The main purpose of this approach is not the visualization of the weather data. The main purpose is to implement both different methodologies as explained before in one application. For this reason, a preprocessing application was developed, which allows to configure and to monitor flexible preprocessing tasks in an easy way. By using a project management system, it is possible to configure and to store different preprocessing tasks settings in a project. Each project consists of the following components:

description: a unique name for identification

mqtt broker: information about the used mqtt broker

mqtt client: information about the mqtt client information

topic: name of the topic, where the data should be subscribed

task: the defined chained task

method: working method (task queue or multiprocessing)

buffering factor: count input data in buffer

arrival rate: time difference between data points to arrive in the buffer

worker count: count of used workers

Figure 5 shows the project overview of the Preprocessing App. This view is the central point of the Preprocessing App. There is an overview about all created projects, where it is possible to go into detail for each project. It is also possible to start and stop the preprocessing for a selected project.

Web sites have a high risk of security issues, especially if they contain input fields. One of the most common issues is cross site scripting. In the background of the industry 4.0 cross site scripting issues (Stock et al. 2017) are most expensive for companies that use web technology especially in their production environment. Each security gap leads to expensive consequences and if it takes a long time to less trust in new technologies. To avoid this risk, the templates from the Preprocessing App were tested with the methods of Stigler et al. (2018) to detect cross site scripting vulnerabilities in websites. All implemented templates are now guarded against cross site scripting attacks. 
Figure 5. Preprocessing App Project Overview

$\because$ Hochschule Aalen

Preprocessing App

E project overview

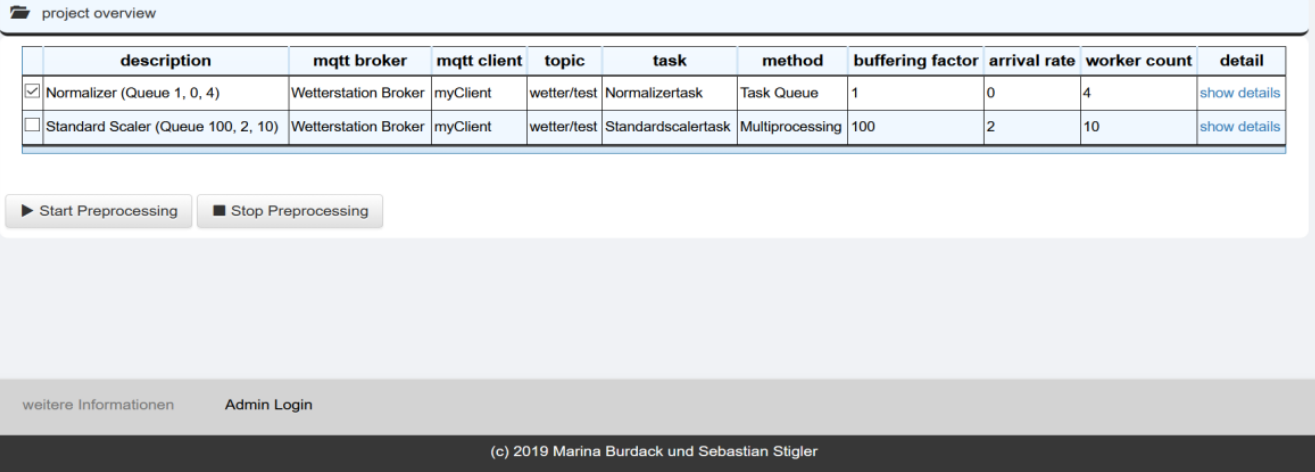

Source: Authors

Figure 6 shows the web template to create new projects over the admin panel from the web application. All input fields are checked for cross site scripting vulnerabilities with the tests from Stigler et al. (2018). The report shows that the Preprocessing App is protected against the most common security flaws.

Figure 6. Add New Project

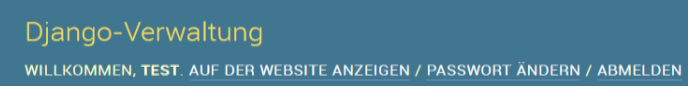

Start : Wetterstation , Projects > project hinzufügen

\section{project hinzufügen}

Description

Normalizer (Queue 1, 0, 4)

MqttBroker:

Wetterstation Broker

Mqttclient:

myclient

subscriber zun at

Task:

Normalizertask

Method:

Task Queue

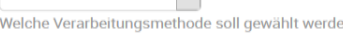

Buffering factor.

$1 \div$

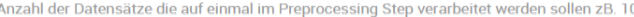

Arrival rate:

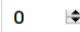

Worker count:

$4 \quad=$

Ersteller-

mariná

Source: Authors. 


\section{Evaluation Data}

The test data set consists of real-time sensor data from the Davis weather station of the Aalen University (http://wetter.infotronik.htw-aalen.de/). This weather station publishes one new message every minute by a MQTT client. Each message consists of a json - structure which contains 26 numerical values like wind speed, outdoor temperature, barometer and seven values as strings, for example the wind direction. A MQTT Broker - in our case mosquitto MQTT manages this data under the topic "'wetter"'. To store these messages another MQTT client subscribes the topic and stores each message as one data row in a Postgres database. At the end of the data collection process the test data set contained 750,000 data rows.

These 750,000 data rows were then packed in different bucket sizes: 1,10 , 100, 1000, 3000, 5000, 7500 and 10000 rows/message.

From now on row or data row denotes a single data package directly out of the data source. When we talk about a message we mean a single data package after the buffering component. A message contains several data rows. The amount is determined by the buffering factor.

For real-time testing, we send the messages from these datasets without delay to the application.

\section{Preprocessing Tasks for Evaluation}

In contrast to the prepare task the duty of the result task depends on what you want to do with the result afterwards: the data could be transformed into a different, converted into a graphic or sent to another application. Therefore in the evaluation this task is only implemented as a dummy task which receives the data and does nothing with it.

Beside these tasks, 14 different classical preprocessing tasks will be implemented in the application. The first six tasks are methods directly from the modern data science library for python, pandas (McKinney et al. 2019). These methods are:

- $a b s$

- aggregated sum

- drop duplicates

- handling missing

- fillna_mean

- fillna_median

- fillna_zeros

In addition eight tasks are implemented from the scikit learn library (Pedregosa et al. 2011, Cournapeau and contriburors 2019). These tasks consist of:

- max_abs_scaler

- min_max_scaler_0_1 
- $\min \_m a x \_s c a l e r \_m 1 \_1$

- normalizer

- quantil_tranformer_normal

- quantil_transformer_uniform

- robust_scaler

- standard_scaler

Evaluating the Service Times for the Various Subtasks and Applying of Queueing Theory

We used a Server from Nvidia for the evaluation with the following specifications:

Nvidia DGX-Station

CPU Intel Xeon(R) CPU E5 2698 v4 @ 2.20GHz x 40 12

RAM 256 GB

$S S D$ 6TB

In the methodology section we provided an inequality to determine under which circumstances the application processes the data without blocking.

An important part of this inequality is the service time $\mu^{-1}$. To determine the service time of each of the preprocessing task we used the following procedure:

1. Choose 75 messages from each bucket of our evaluation data.

2. Send a message to be processed by the chosen task and execution method (single threaded, multiprocessing and distributed task queue).

Measure the execution time.

3. Repeat until all messages where processed.

We use the single threaded approach to get the pure execution time of the task without any overhead from the multiprocessing resp. the distributed task queue approach.

This overhead in the multiprocessing case is the sending of the data from the main process to one of the worker in the worker pool and the time it takes to send the result back. In the case of the distributed task queue the overhead contains the sending of the serializes pandas data frame to the worker node via Celery (with the help of Redis) and the deserialization back to a pandas data frame so that the task can do its job. Furthermore the result must be serialized as well and then sent, via the Celery infrastructure, back to the main process.

As you can see, the overhead of the distributed task queue per message is higher than the overhead of the multiprocessing approach. And so the old proverb "There is no such thing as free lunch" is again confirmed. If you want to increase the processing power of your system by switching from multiprocessing to a

\footnotetext{
${ }^{12} 20$ physical Cores (40 with Hyper Treading).
} 
distributed task queue, you have to pay with an increased transportation time of the data. We will later show how to tune the system to compensate this increase in the overall service time.

Figure 7 shows the result of the evaluation procedure from above, applied to the prepare task, run in single threaded mode. The first column shows the size of the messages. For example the last row indicates the test set where each of the 75 messages contains 10000 data rows. A row contains the mean (depict with $\varnothing$ ) and the standard deviation (depict with $\sigma$ ) of the service time for the processing of the whole message in $\mu s$ and the service time per data row.

Figure 7. Table of Single Threaded Approach for the Task_Prepare (Henceforth known as Base Line)

\begin{tabular}{lrrrr} 
& \multicolumn{2}{c}{ duration/msg } & \multicolumn{2}{c}{ duration/row } \\
rows/msg & \multicolumn{1}{c}{$\sigma$} & \multicolumn{1}{c}{$\sigma$} & \multicolumn{1}{c}{$\sigma$} & \multicolumn{1}{c}{$\sigma$} \\
$\mathbf{1}$ & 3840.881 & 453.105 & 3840.881 & 453.105 \\
$\mathbf{1 0}$ & 4019.249 & 217.332 & 401.925 & 21.733 \\
$\mathbf{1 0 0}$ & 5951.921 & 413.597 & 59.519 & 4.136 \\
$\mathbf{1 0 0 0}$ & 23652.684 & 1825.831 & 23.653 & 1.826 \\
$\mathbf{3 0 0 0}$ & 63629.374 & 5091.716 & 21.210 & 1.697 \\
$\mathbf{5 0 0 0}$ & 103221.863 & 8230.345 & 20.644 & 1.646 \\
$\mathbf{7 5 0 0}$ & 156601.112 & 12795.871 & 20.880 & 1.706 \\
$\mathbf{1 0 0 0 0}$ & 207001.974 & 15776.564 & 20.700 & 1.578 \\
\multicolumn{2}{l}{ Source: Authors. } & & &
\end{tabular}

Figures 8 and 9 show the result of this evaluation of all preprocessing tasks. Each subplot has logarithmic $\mathrm{x}$ and $\mathrm{y}$ axis. The $\mathrm{x}$ axis denotes the number of data rows per message. In Figure 8 the y axis is the service time per message in $\mu s$ and in Figure 9 it is the service time per data row in $\mu s$.

Each subplot shows a graph for the base line, the multiprocessing variant and the task queue variant. It can easily be seen, that using the base line offers the best service time, followed by multiprocessing and the task queue approach offers the worst service time. This disadvantage can be compensated by the fact, that multiprocessing can use more than one CPU core to process tasks in parallel compared to the base line implementation; but it can only use the cores on one single physical computer. A task queue can spread the tasks over all the cores on multiple computers.

The dark grey stripe on the right of each subplot denotes a region where the slope of each curve increased significantly. This happened because the processing of the task with such a large message reaches (or at least is near) the limits of the CPU. You should avoid using messages of this size.

Figure 8 shows, that up to $100 \frac{\mathrm{rows}}{\mathrm{msg}}$, the service time per message, stays roughly the same. The time doubles at most while the number of rows per message grows by a factor of a hundred. And in the interval from 1 to $1000 \frac{\text { rows }}{\mathrm{msg}}$ the time increases by a factor less than 10 . Therefore the length of message has not a very high impact on the time to process it. 
Figure 9 shows the advantage of buffering more data rows into a single message to process it in one go: In a message with many data rows, the service time for each data row in it decreases significantly.

Figure 8. Mean Service Time per Message
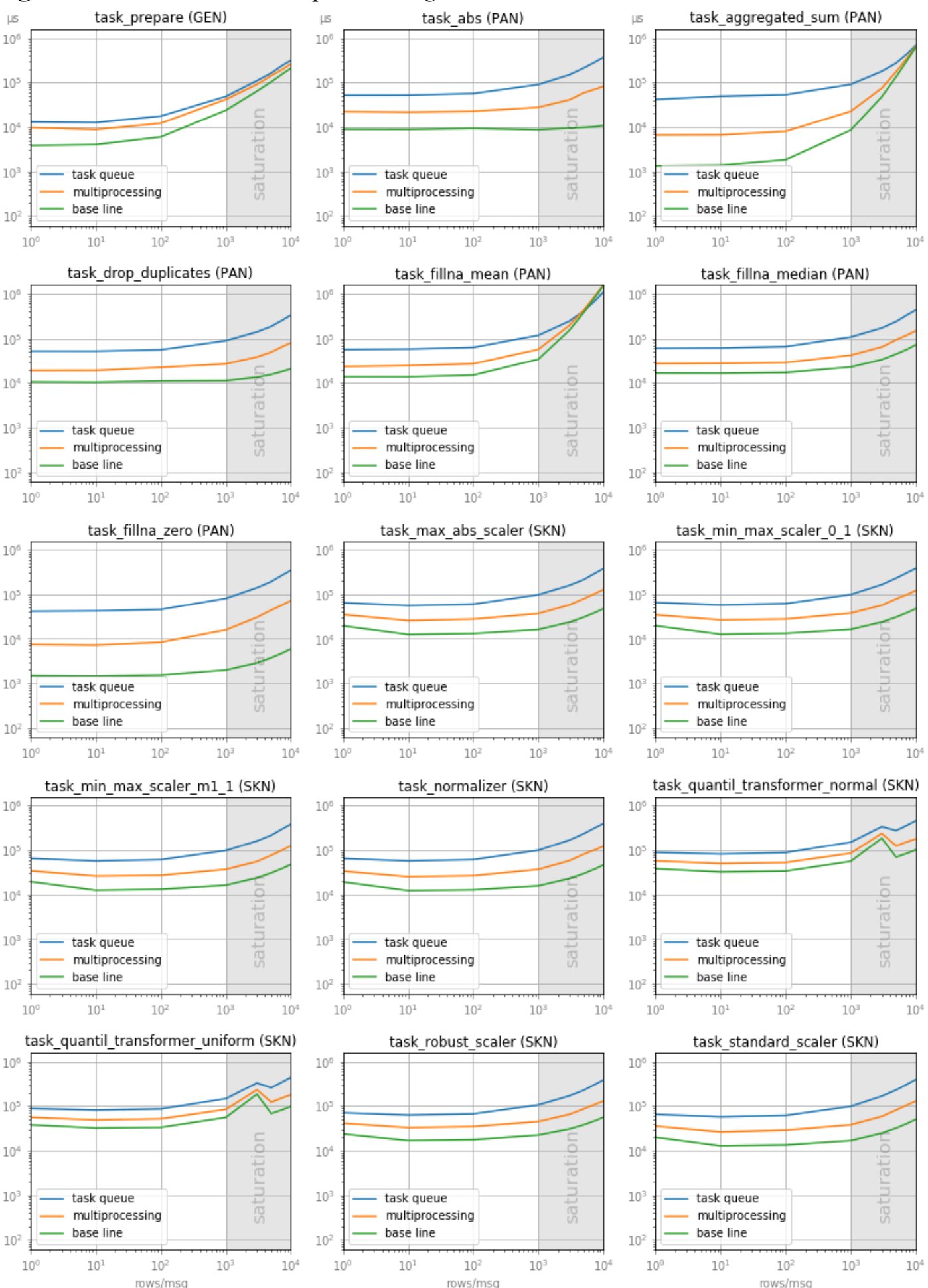

Source: Authors. 
Vol. 7, No. $1 \quad$ Stigler \& Burdack: A Practical Approach of Different Programming ...

Figure 9. Mean Service Time per Row
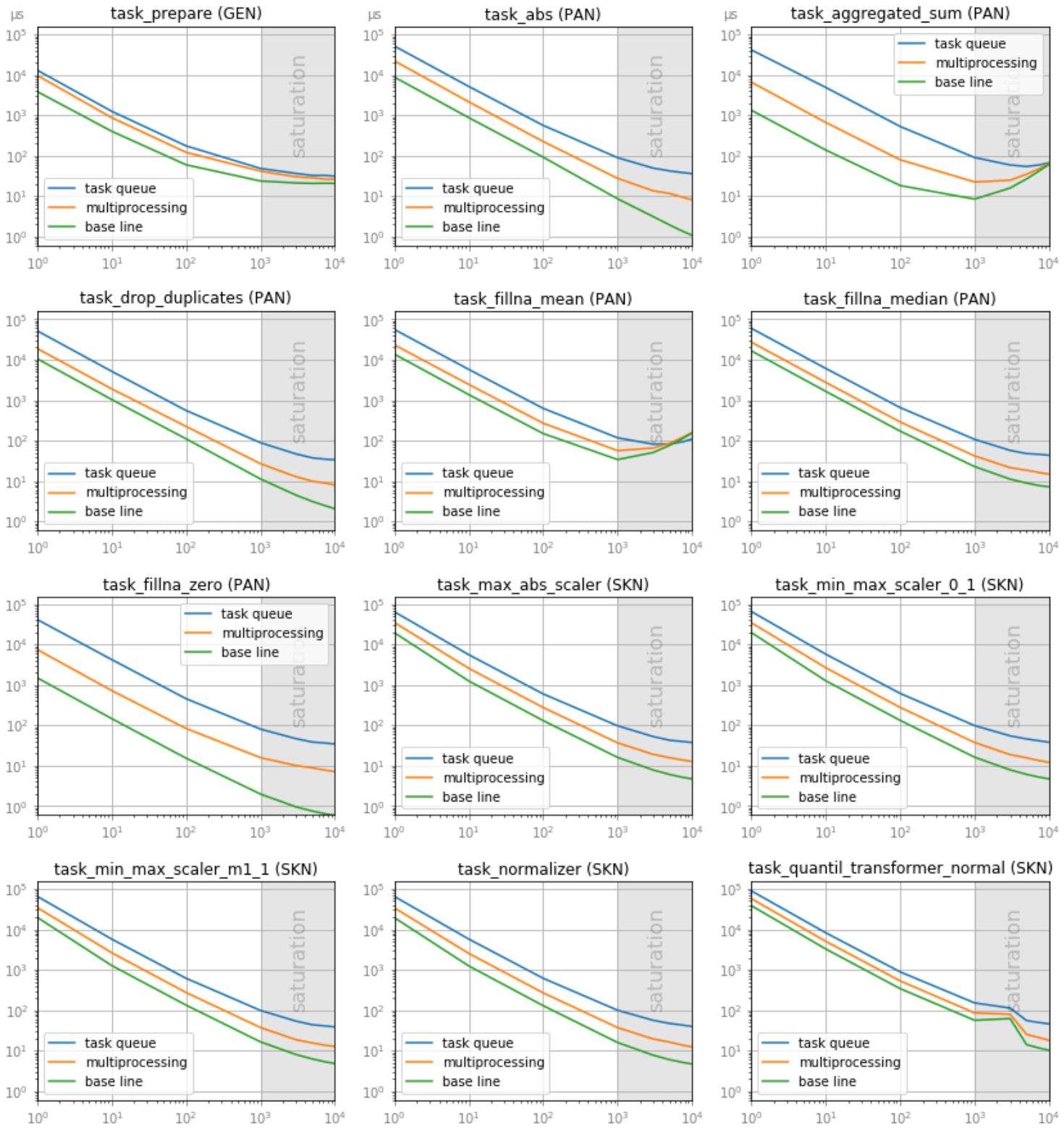

task_quantil_transformer_normal (SKN)

task_quantil transformer_uniform (SKN)
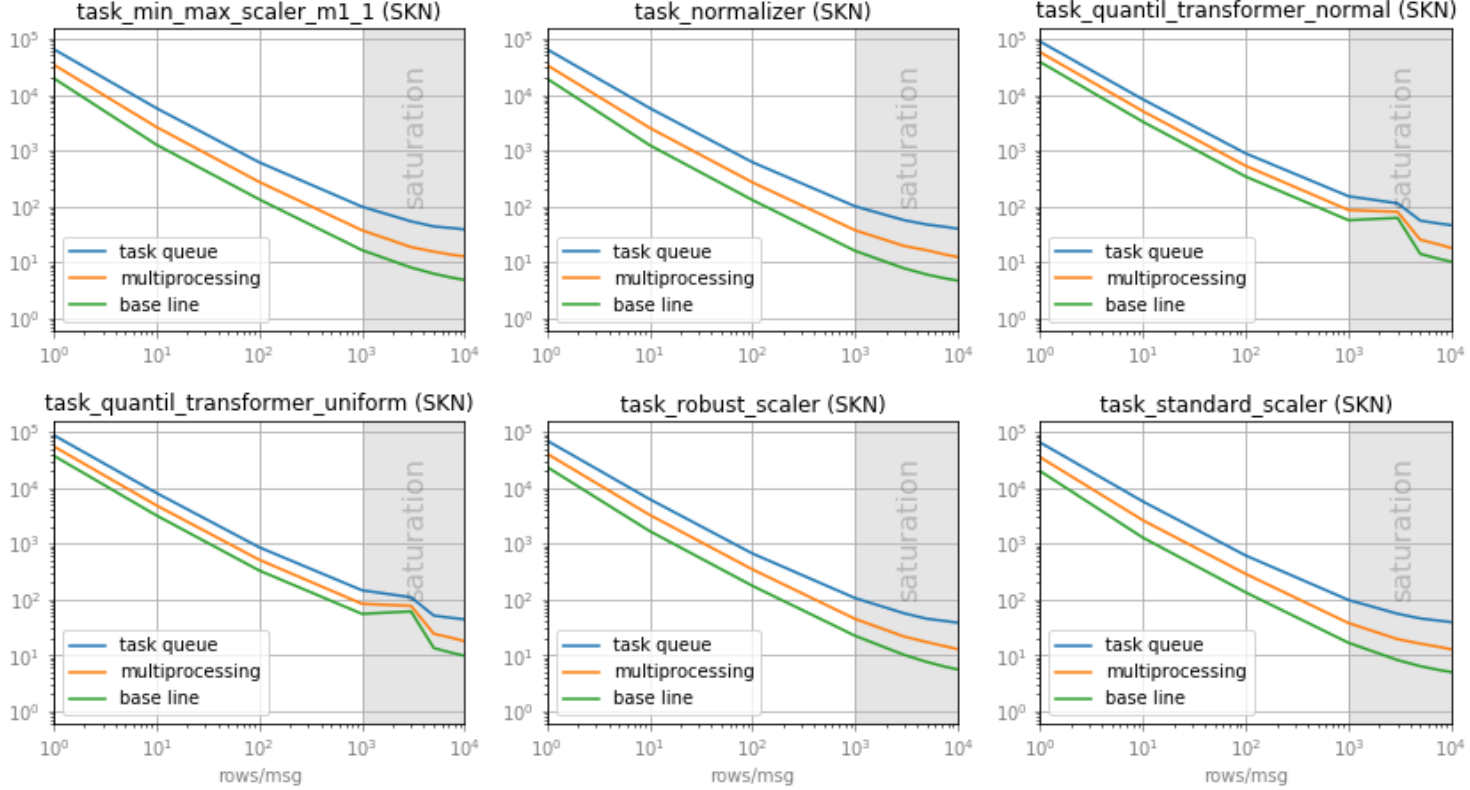

Source: Authors. 


\section{Discussion}

How can we Use this Data to Scale our Application Properly

For the purpose of this example calculation we assume that there are neither restrictions on an acceptable arrival rate nor constraints regarding the delay the buffering will introduce to the system by waiting until the predefined number of data rows were received from the buffer before sending a message into the application.

How to Keep the Service Time Constant while Switching from Multiprocessing to Task Queues?

Assume you use the task_fillna_zero task and use no buffering in multiprocessing mode. If you look in Figure 9 at the subplot of this task you can see, that its service time for multiprocessing is approximately $7 \mathrm{~ms}$. On the curve for the task queue, in the same subplot, you get this service time if you buffer with approximately 6 rows per message.

What can be Done if the Arrival Rate Increases but the Service Time should Kept the Same in a Task Queue?

In this case you can use the inequality for the server utilization $\rho$. If you increase the arrival rate by the factor of $n$ then the number of services $c$ has to grow with the same factor.

How to Use the Information from Above to Determine Good Parameter for a Given Chained Task?

In the central subplot of Figure 10 we show the mean service time per data row of the following chained task ${ }^{13}$ (with the approximate service times at a buffer factor of 10 on a task queue):

1. task_prepare $\mu_{p}^{-1} \approx 1.3 \mathrm{~ms}$

2. task_fillna_zero $\mu_{f}^{-1} \approx 4.2 \mathrm{~ms}$

3. task_normalizer $\mu_{n}^{-1} \approx 5.7 \mathrm{~ms}$

4. task_drop_duplicates $\mu_{d}^{-1} \approx 5.1 \mathrm{~ms}$

The complete chained task should therefore have a service time of approximately $16.3 \mathrm{~ms}$.

Now we will use the inequality for the server utilization to calculate an arrival rate, with respect to a stable system $(\rho<1)$. In the beginning of this section we

\footnotetext{
${ }^{13}$ We ignore the service time for the task_result in this example as we don't want to use the processed data afterwards! In a real application, the service time for the task_result must be added, too.
} 
specified our test server as a server with 20 CPU cores, therefore we use $c=20$ in the inequality. As we used service time per data row, we want to calculate $\lambda_{d s}$ (the arrival rate measured at the data source).

$$
\lambda_{d s}<c \mu=20 \cdot \frac{1}{16.3 m s}=1226.99 \mathrm{~Hz}
$$

To determine the delay between two consecutive data rows we need to build the inverse of $\lambda_{d s}$ :

$$
\lambda_{d s}^{-1}>\frac{1}{1226.99 \mathrm{~Hz}}=815 \mu \mathrm{s}
$$

To be on the save side, we will use $\lambda_{d s}^{-1}=900 \mu s$.

Figure 10. Mean Service Time per Data Row of the Above Chained Task (The left subplot shows the result, when the data rows are sent with no delay ${ }^{14}$ to the chained task. The central plot shows the sum of subplots in Figure 9 that forms the subtasks of the chained task. The right subplot is the result of the test run with $\lambda_{d s}^{-1}=0.0009 s$ ).
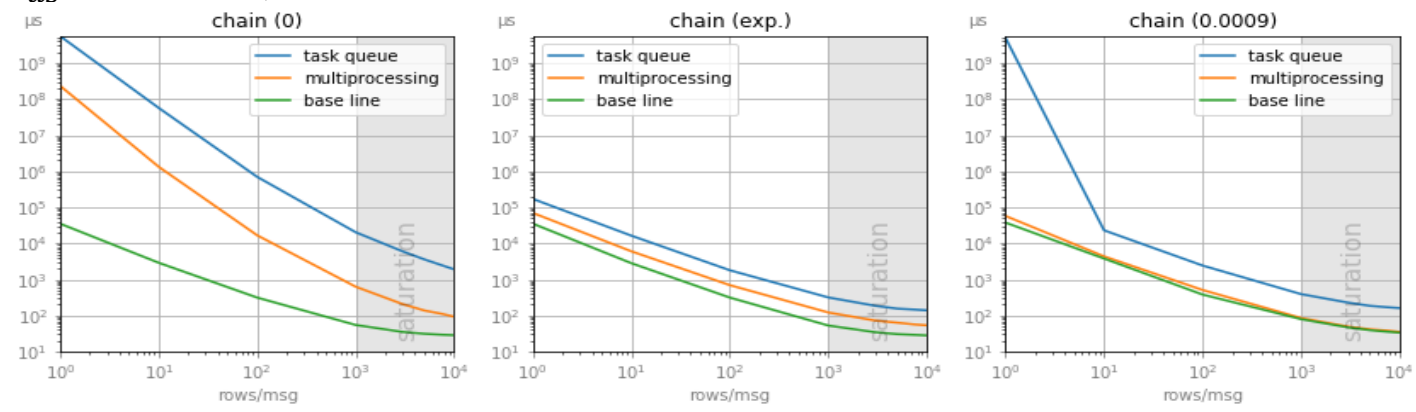

Source: Authors.

The data for the left and right subplot of Figure 10 was created by sending the complete evaluation data without delay to the application on the DGX-Station, i.e. we send 750,000 data rows in bucket from 1 row per message up to 10000 rows per message to the chained task.

In subplot "chain (0.0009)"' of Figure 10 the base line matches the prediction in "chain (exp.)" quite nicely. The service time for the multiprocessing is even a bit better than the prediction. The graph for the task queue shows that the prediction from $10 \frac{\text { rows }}{\mathrm{msg}}$ onwards are a bit worse than the prediction. For the $1 \frac{\text { rows }}{\mathrm{msg}}$ entry we see a very high value for the service time. But this is ok as we calculated the arrival rate based on the values of the task queue at $10 \frac{\mathrm{rows}}{\mathrm{msg}}$.

To verify whether we produced a stable configuration, we have to calculate the server utilization corresponding to the plots in Figure 10.

\footnotetext{
${ }^{14}$ We measured $4 \mu s$ between any two consecutive data rows.
} 
Figure 11. Server Utilization (The first three columns show the utilization of the chained task for the base line (bl), the multiprocessing (mp) and the task queue (tq) variant of the software while serving new data rows with no delay. The three columns in the middle show the expected utilization with $\lambda_{d s}^{-1}=0.9 \mathrm{~ms}, c=20$ for multiprocessing and task queue and $c=1$ for the base line $e^{15}$ and the service time according to the summed service time of the above named tasks depicted as in the central subplot of Figure 10. The last three columns show the actual run of this chained task on the DGX-Station Server).

\begin{tabular}{|c|c|c|c|c|c|c|c|c|c|}
\hline \multirow[b]{2}{*}{ rows $/ \mathrm{msg}$} & \multicolumn{3}{|c|}{ chain (0) } & \multicolumn{3}{|c|}{ chain (exp.) } & \multicolumn{3}{|c|}{ chain (0.0009) } \\
\hline & $\mathrm{bl}$ & $\mathrm{mp}$ & tq & bl & $\mathrm{mp}$ & tq & bl & $\mathrm{mp}$ & tq \\
\hline 1 & 8756.157 & 2897599.497 & 69401701.487 & 39.026 & 3.872 & 9.474 & 41.771 & 3.191 & 275433.419 \\
\hline 10 & 723.311 & 16593.341 & 712226.313 & 3.127 & 0.334 & 0.907 & 4.209 & 0.242 & 1.277 \\
\hline 100 & 78.269 & 208.676 & 8716.986 & 0.348 & 0.039 & 0.100 & 0.423 & 0.028 & 0.135 \\
\hline 1000 & 13.629 & 7.847 & 254.033 & 0.058 & 0.007 & 0.018 & 0.088 & 0.005 & 0.022 \\
\hline 3000 & .736 & 2.578 & 74.856 & 0.038 & 0.004 & 0.010 & 0.052 & 0.003 & 0.013 \\
\hline 5000 & .773 & 1.712 & 44.746 & 0.034 & 0.004 & 0.009 & 0.044 & 0.002 & 0.010 \\
\hline 7500 & 7.317 & 1.388 & 30.874 & 0.032 & 0.003 & 0.008 & 0.039 & 0.002 & 0.009 \\
\hline 10000 & 7.099 & 1.153 & 23.754 & 0.031 & 0.003 & 0.008 & 0.037 & 0.002 & 0.009 \\
\hline
\end{tabular}

Source: Authors.

It can be seen, that the $\rho$ values for all implementation types of the prediction (central three columns) in the $1 \frac{\text { rows }}{\mathrm{msg}}$ as well as the base line value for $10 \frac{\mathrm{rows}}{\mathrm{msg}}$ should lead to instabilities as the $\rho$ values are strictly greater than one. All other entries indicate that the resulting run should lead to stable executions of the chained task. Although the $90.7 \%$ utilization for the task queue at $10 \frac{\text { rows }}{\mathrm{msg}}$ should lead to a stable process, as Figure 11 shows, the task queue performs a little worse than predicted, which actually leads to an instable process.

If we compare our expectations with the actual runs (three rightmost columns) we see, that with the exception of the $10 \frac{\text { rows }}{\mathrm{msg}}$ all predictions hold.

We suspect, that the utilization of the network connection to the worker node may be the reason for the results of the task queue. The reason for this assumption lies in the construction of the data for Figure 9: We sent one message and then measured the time until the result was ready before we sent the next message. For the calculation of the task queue values for Figure 10 "chain (0.0009)" we sent the messages with a constant rate of $1111,11 \mathrm{~Hz}\left(\frac{1}{900 \mathrm{~ms}}\right)$ over the network to the worker node.

Finally we have to discuss the leftmost subplot of Figure 10 resp. the three leftmost columns of the table in Figure 11.

Here we want to show how an instable process may look like. The slope of the multiprocessing and the task queue graph in Figure 10 "chain (0)" is much steeper than the slope of the base line. In contrast, the slopes in "chain (exp.)" of the three graphs are very similar and when compared to the stable parts of the graph in "chain (0.0009)" it can be seen, that the slops in this areas are similar too. The values in the table in Figure 11 show, with $\rho$ values much greater than one, that the graphs actually show instable processes.

\footnotetext{
${ }^{15}$ The base line is a single threaded implementation in Python. Hence only one core can be used (Python Software Foundation 2019).
} 


\section{Some Advice on Choosing the Architecture for your Own Application}

The inequality for the server utilization can help you to determine the number of servers $c$ so that the system stays stable.

If $c$ is less or equal to number of cores on the host server then multiprocessing should be used.

If $c$ exceeds the number of cores on the host system then it is advisable to use a distributed task queue with at least $c$ task queue worker processes.

\section{Conclusions}

This article demonstrated how far you can get in a data processing application with Python libraries only. Furthermore, it was demonstrated that even if Python has its flaws in puncto utilization of more than on core per process (Python Software Foundation 2019), it is possible to write an application that circumvented this problem by using multiprocessing or even a distributed task queue to utilize more than one server for the data processing.

The architecture of the application for the multiprocessing and the task queue approach was presented and it was shown how to organize a data processing task that is built from some basic data processing tasks and how to chain them together.

An essential part of this article was the use of queueing theory to explain how to scale an application that works as a data processing pipeline.

In the findings section, it was described how the application works and the evaluation of the performance of the two aspects of the application (the multiprocessing and the task queue aspect) was discussed.

Finally, it was concluded that the restriction to typical preprocessing tasks was not as severe as it seems. The techniques to determine the utilization of a server can be directly applied to machine learning tasks and post processing task like visualization.

\section{References}

Babuji YN, Chard K, Foster IT, Katz DS, Wilde M, Woodard A, Wozniak JM (2018) Parsl: scalable parallel scripting in python. $10^{\text {th }}$ International Workshop on Science Gateways, 13-15 June 2018.

Bhat UN (2015) An introduction to queueing theory. Modelling and analysis in applications. Basel: Birkhäuser.

Borthakur D, Gray J, Sarma JS, Muthukkaruppan K, Spiegelberg N, Kuang H, ... Aiyer A (2011) Apache hadoop goes realtime at facebook. In Proceedings of the 2011 ACM Sigmod International Conference on Management of Data, 1071-1080. New York, USA: ACM.

Burdack M, Rössle M, Kübler R (2018) A concept of an in-memory database for iot sensor data. Athens Journal of Sciences 5(4): 355-374. 
Burdack M, Rössle M (2019) A concept of an interactive web-based machine learning tool for individual machine and production monitoring. In Intelligent Decision Technologies, 183-193. Singapore: Springer Singapore.

Cournapeau D and contriburors (2019) Scikit-learn 0.20.3. Retrieved from https://scikitlearn.org.

Fein P (2018) Concurrency. Retrieved from https://wiki.python.org/moin/Concurrency.

Kaggle, Crawford C, Montoya A, O'Connell M, Mooney P (2018) 2018 kaggle $m l \& d s$ survey. The most comprehensive datdata available on the state of $\mathrm{ml}$ and data science. Retrieved from https://www.kaggle.com/kaggle/kaggle-survey-2018.

Karau H (2015) Learning spark - lightning-fast big data analysis. Sebastopol: O’Reilly Media, Inc.

Kotliar, M., Kartashov, A., \& Barski, A. (2018). CWL-airflow: A lightweight pipeline manager supporting common workflow language. bioRxiv, 249243.

Lam C (2010) Hadoop in action ( $1^{\text {st }}$ Edition). Greenwich, CT, USA: Manning Publications Co.

Lunacek M, Braden J, Hauser T (2013) The scaling of many-task computing approaches in python on cluster supercomputers. In 2013 IEEE International Conference on Cluster Computing, 1-8. https://doi.org/10.1109/CLUSTER.2013.6702678.

McKinney W, Augspurger T, Ayd W, Bartak C, Battiston P, Cloud P, ... Young G (2019) Pandas. Python data analysis library v0.24.2. Retrieved from https://pandas. pydata.org/.

Meng X, Bradley J, Yavuz B, Sparks E, Venkataraman S, Liu D, Freeman J, Tsai DB, Made M, Owen S, Xin D, Xin R, Franklin MJ, Zadeh R, Zaharia M, Talwalkar A (2016) Mllib: Machine learning in apache spark. The Journal of Machine Learning Research 17(34): 1-7.

Pedregosa F, Varoquaux G, Gramfort A, Michel V, Thirion B, Grisel O, Blondel M, Prettenhofer P, Weiss R, Dubourg V, Vanderplas J, Passos A, Cournapeau D, Brucher M, Perrot M, Duchesnay É (2011) Scikit-learn: Machine learning in python. The Journal of Machine Learning Research 12: 2825-2830.

Pivotal Software Inc. (2019) RabbitMQ. Retrieved from https://www.rabbitmq.com/.

Python Software Foundation and JetBrains (2018) Python developers survey 2018 results. Retrieved from https://www.jetbrains.com/research/python-developers-survey-2018.

Python Software Foundation. (2019) Thread state and the global interpreter lock. Retrieved from https://docs.python.org/3/c-api/init.html\#thread-state-and-the-globalinterpreter-lock.

Rössle M, Kübler R (2017) Quality prediction on die cast sensor data. In ATINER's Conference Paper Series, number: COM2017-2272. Athens: ATINER.

Sanfilippo S and contriburors (2019) Redis 5.0.4. Retrieved from https://redis.io.

Solem A and contributors (2019) Celery: Distributed task queue 4.3.0. Retrieved from http://www.celeryproject.org.

Stigler S, Karzhaubekova G, Karg C (2018) An approach for the automated detection of xss vulnerabilities in web templates. Athens Journal of Sciences 5(3): 261-280.

Tanenbaum AS, Bos H (2014) Modern operating systems (4 ${ }^{\text {th }}$ Edition). Upper Saddle River, NJ, USA: Prentice Hall Press.

Van der Stock A, Smithline N, Gigler T (2017) OWASP top 10 - 2017. The ten most critical web application security risks. Retrieved from https://bit.ly/2qK2CcE.

Vincent WS (2018) Django for beginners. Build websites with python \& django. SMTEBOOKS.

Vinjumur JK., Becker E, Ferdous S, Galatas G, Makedon F (2010) Web based medicine intake tracking application. In Proceedings of the $3^{\text {rd }}$ international conference on 
Vol. 7, No. $1 \quad$ Stigler \& Burdack: A Practical Approach of Different Programming ... pervasive technologies related to assistive environments, 37:1-37:8). New York, USA: ACM.

Wirth R, Hipp J (2000) CRISP-dm: Towards a standard process model for data mining. In Proceedings of the fourth international conference on the practical application of knowledge discovery and data mining, 29-39. Retrieved from https://bit.ly/2ONx0e7. Wouters T (2017) GlobalInterpreterLock. Retrieved from https://bit.ly/2OMEo9I. 